\title{
Wide wavelength tuning of sampled grating tunable twin-guide laser diodes
}

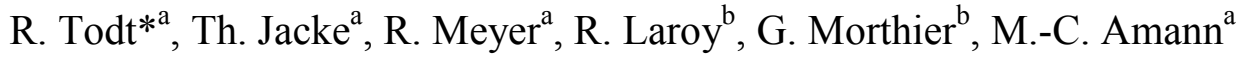 \\ ${ }^{\text {a }}$ Walter Schottky Institute, Technical University of Munich, D-85748 Garching, Germany \\ ${ }^{\mathrm{b}}$ Department of Information Technology, Ghent University-IMEC, B-9000 Ghent, Belgium
}

\begin{abstract}
Tunable laser diodes with a tuning range of several tens of nanometers are generally being acknowledged as key components for future generation optical networks. However, all presently available devices suffer from several serious drawbacks. The most well-known issue is the time-consuming calibration procedure that has to be carried out for every single device.

Recently the so-called sampled or superstructure grating tunable twin-guide or (S)SG-TTG laser diode has been suggested to overcome some of the prevailing problems. In this paper we will present tuning characteristics of first devices and discuss the influence of facet reflections on the tuning behaviour.

The widely tunable twin-guide laser diodes operate around $1.55 \mu \mathrm{m}$ and have a continuous tuning range of $\sim 2 \mathrm{~nm}$. However, by utilizing Vernier-effect tuning, the overall quasi-continuous tuning range is extended to $28 \mathrm{~nm}$. Within this tuning range, five supermodes can be continuously tuned without the occurrence of any mode hops. The side-mode suppression ratio is kept between 25 and $37 \mathrm{~dB}$.
\end{abstract}

Keywords: semiconductor lasers, tunable lasers, wide wavelength tuning, Vernier-effect tuning, sampled gratings, superstructure gratings

\section{INTRODUCTION}

There has been considerable interest in monolithic, edge-emitting, electronically tunable laser diodes with a tuning range of several tens of nanometers over the last couple of years, which was mainly driven by the broad field of application for these widely tunable lasers. They are regarded as key components for future fiber optical communication networks ${ }^{1}$, enabling new network concepts like optical packet or burst switching, label switching or bandwidth on demand, just to name a few. Besides this, they are also highly attractive light sources for gas sensing applications, ${ }^{2}$ for fiber sensor interrogation ${ }^{3}$ as well as for optical coherence tomography. ${ }^{4}$

First widely tunable lasers have already been presented more than a decade ago. However, only a very limited amount of device types has been developed since then (an overview can be found in Refs. 5,6), and practically all of the presently available types still suffer from several serious drawbacks. For example device characterization is a very common issue: since typically three or even more tuning currents are required to set the emission wavelength, the device calibration that is required for every single laser diode becomes time-consuming and, therefore, also expensive. Further drawbacks include limited output power, limited direct modulation capabilities and fabrication complexity.

Only recently, the so-called sampled or superstructure grating tunable twin-guide or (S)SG-TTG laser diode has been suggested. $^{7}$ This novel widely tunable laser is based on the tunable twin-guide laser with distributed feedback (DFB$\mathrm{TTG}^{8}$ and is essentially a two-section phase-shifted DFB laser. Since a phase tuning section is not necessary in this device design, only two tuning currents are required to achieve wide quasi-continuous wavelength tuning. This facilitates fast device characterization and easy device control. Furthermore the DFB-like nature of the device is expected to result in high side-mode rejection. Additionally, there is potential for high power operation and even for direct modulation up to high frequencies of $10 \mathrm{GHz}$ and more.

In this paper we will present tuning characteristics of first devices and qualitatively discuss the influence of facet reflections on the tuning behaviour of the laser.

* e-mail: todt@wsi.tum.de; phone: +49-(0)89-289 12767; fax: +49-(0)89-320 6620; www.wsi.tum.de 


\section{OPERATION PRINCIPLE}

The tunable twin-guide laser with sampled or superstructure gratings is based on the tunable twin-guide laser with distributed feedback (DFB-TTG), but employs two tuning sections with sampled gratings (SGs) or superstructure gratings (SSGs) for Vernier-effect tuning instead of an DFB grating. Schematic drawings of the device are depicted in Fig. 1.

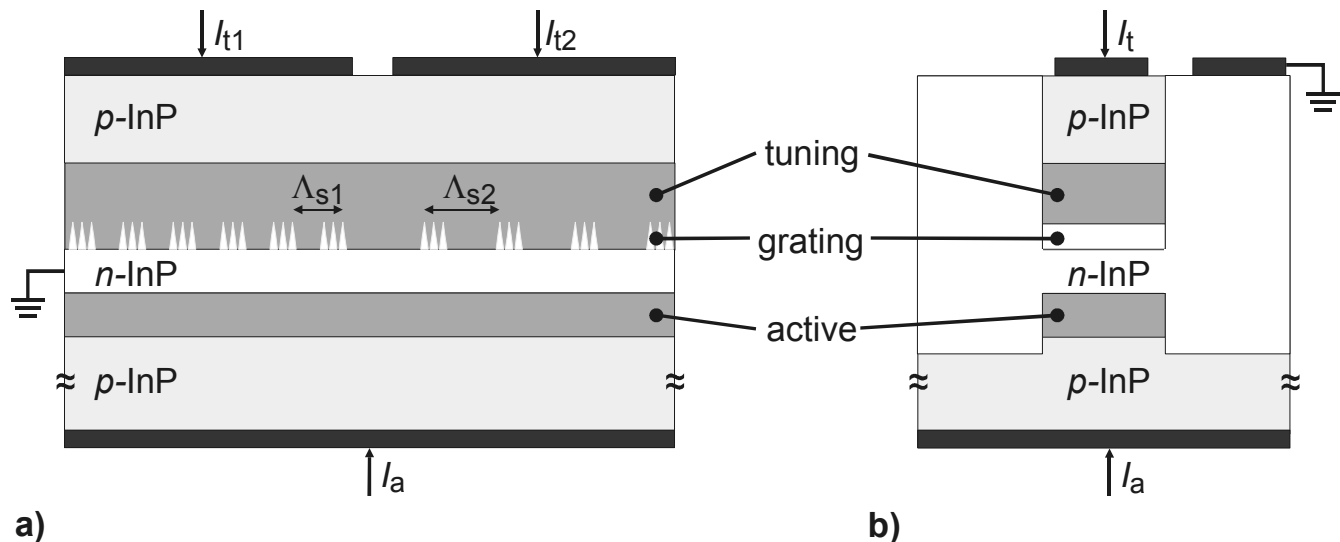

Fig. 1. Schematic drawings of a widely tunable SG-TTG laser diode.

a) longitudinal cross-section, b) transverse cross-section.

One of the main features of this design is the vertical integration of active layer, tuning layer and gratings. From an optical point of view, the device structure forms a single-mode waveguide, whose effective refractive index can be changed by current injection into the tuning layer. Longitudinal single-mode operation is achieved by the use of (S)SGs, which not only provide reflection at the Bragg wavelength (as would be the case for an DFB grating) but provide a comb-like reflection spectrum (Fig. 2a). The reflection peak spacing is determined by the sampling or superstructure period $\Lambda_{\mathrm{s} 1,2}$, which is slightly different for the gratings of tuning section 1 and 2 . Therefore, Vernier-effect tuning ${ }^{9}$ can be employed to overcome the tuning limit that is imposed by the maximum achievable refractive index change. Lasing automatically occurs at the wavelength where reflection peaks from both sections overlap (Fig. 2b). Hence, an additional phase tuning section, which is usually required to adjust the position of the cavity mode in longitudinally integrated DBR-type lasers, is needless.
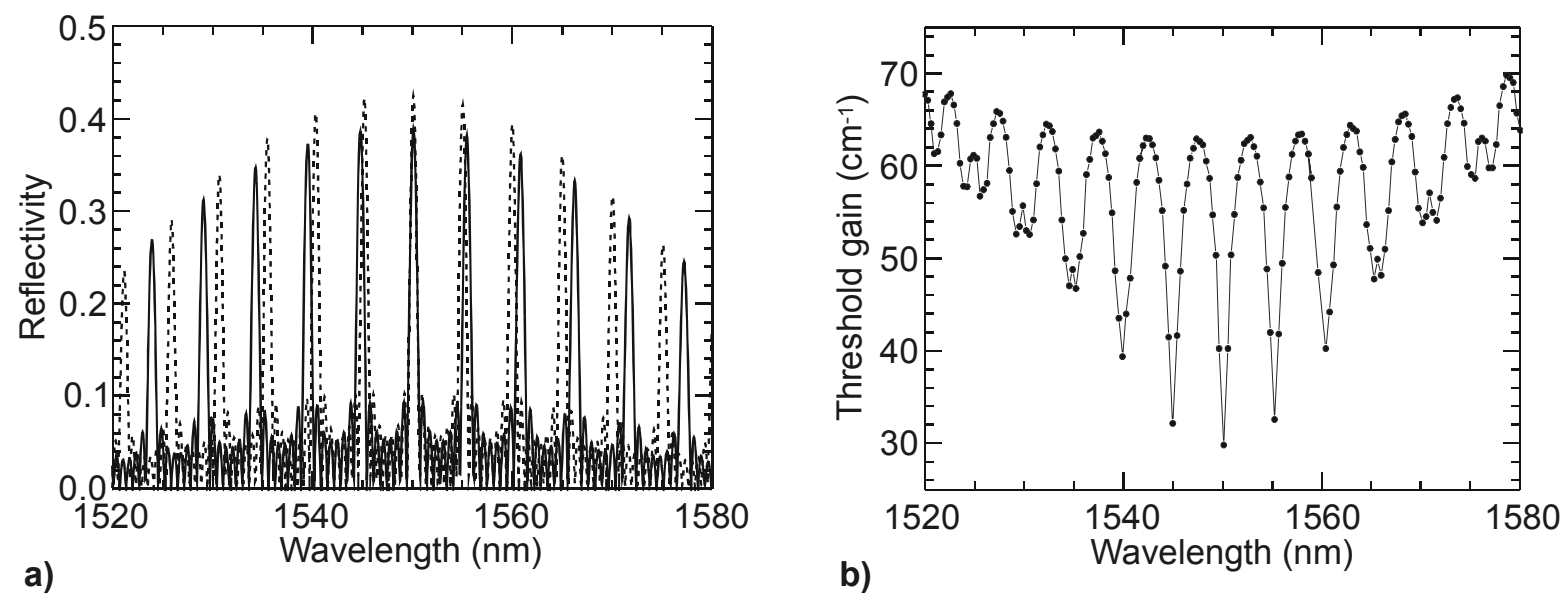

Fig. 2. a) Calculated reflection spectra of SGs with slightly different sampling periods. b) Cavity modes of the corresponding SG-TTGlaser diode (dots indicate the position of the cavity modes, lines are only shown as a guide to the eye). 
As a result, quasi-continuous wavelength coverage with high side-mode suppression is achieved with only two tuning currents, which essentially facilitates fast characterization. Tuning of only one section leads to a transition from one pair of reflection peaks to another and, thereby, to a large wavelength jump, a so-called supermode hop. On the other hand, both sections can also be tuned simultaneously in such a way that the reflection peaks keep on overlapping and one obtains continuous wavelength tuning.

The tuning behavior of the (S)SG-TTG laser significantly differs from previously presented widely tunable multisection DFB lasers with (S)SGs. ${ }^{10}$ Although the device structures are similar at first glance, they are completely different from an electrical point of view. The tuning sections in an (S)SG-TTG laser are electrically decoupled from the active region of the device and, therefore, any arbitrary combination of wavelength shifts of the two (S)SG reflection spectra is obtainable, which is absolutely necessary to achieve full wavelength coverage. In contrast, in a widely tunable multisection DFB laser, tuning is brought about by changing the carrier density inside the active region. However, due to gain-clamping, the refractive indices of both sections cannot be adjusted independently from each other and, therefore, only certain discrete wavelengths are obtainable.

Besides the aforementioned points, one should also mention that the devices can be rather short. In fact, the results presented in this paper are from $600 \mu \mathrm{m}$ long devices, which is about a factor of $2-3$ shorter in comparison to other monolithic widely tunable lasers.

\section{INFLUENCE OF FACET REFLECTIONS}

In the previous description of the device principle it has been implicitly assumed that the facets of lasers are perfectly anti-reflection (AR) coated. There will, however, always be some residual reflections from the facets in a real device. In this section we will therefore discuss what impact facet reflections will have on the (S)SG-TTG laser.

To illustrate the influence of the facet reflections, reflection spectra of a single SG (which basically makes up half of the laser length) have been simulated using a transfer matrix method. The geometry is schematically depicted in Fig. 3. The

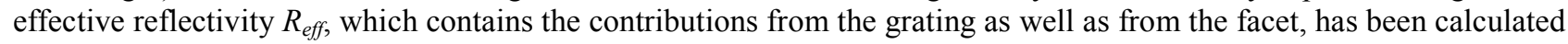
for various facet reflectivities $R_{f}$ and various facet positions (which are denoted as distance $L$ relative to the last grating burst).

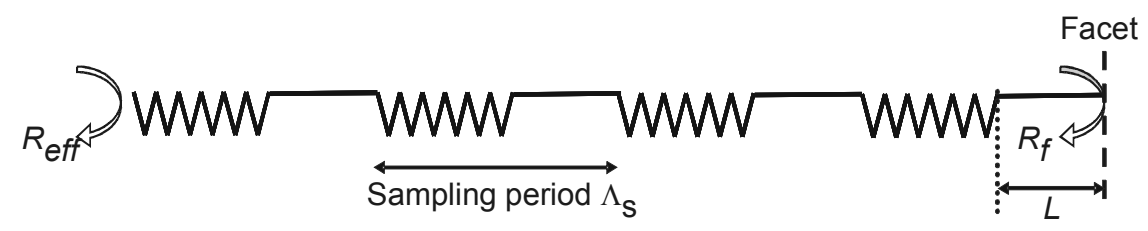

Fig. 3. The effective reflectivity $R_{\text {eff }}$ of one grating section is determined by the interference of reflections from the sampled gratings and from the facet, which is situated a distance $L$ away from the grating.

Results of these simulations are shown in Fig. 4. In both graphs, the case of the perfectly AR-coated facet $\left(R_{f}=0\right)$ is shown as solid line. First of all, Fig. 4a shows how the spacing $L$ between facet and grating influences the reflection spectrum of the SG. For these simulations the power reflectivity of the facet was assumed to be $1 \%$, which is a value that is commonly achieved with single layer AR-coatings. The envelope of SG becomes modulated by interference oscillations that are due to the cavity that is formed by the last grating and the facet. Hence, the strongest distortions are to be expected when the spacing $L$ is on the order of the sampling period, since the oscillation period of the interference pattern will in this case be comparable to the reflection peak spacing of the SG. The sampling period $\Lambda_{\mathrm{s}}$ of the SG was chosen to be $\sim 60 \mu \mathrm{m}$. Thus, with $\mathrm{L} \sim 30 \mu \mathrm{m}$ (shown as dotted line in Fig $4 \mathrm{a}$ ), the oscillation period of the interference pattern is just twice as large as the reflection peak spacing of the SG and, therefore, the reflectivity of one half of the reflection peaks becomes enhanced while it is decreased for the other half. It should be clear that such a reflection spectrum is not well suited to obtain a regular tuning characteristic.

Furthermore it should be noted that the effect of the facet reflections is not only to increase or decrease the reflectivity of the SG reflection peaks. In fact, also the peak positions are shifted by small amounts, as can for example be seen from the reflection peaks at $1576 \mathrm{~nm}$ in Fig 4a. Even such small shifts can readily result in an irregular tuning behaviour. 

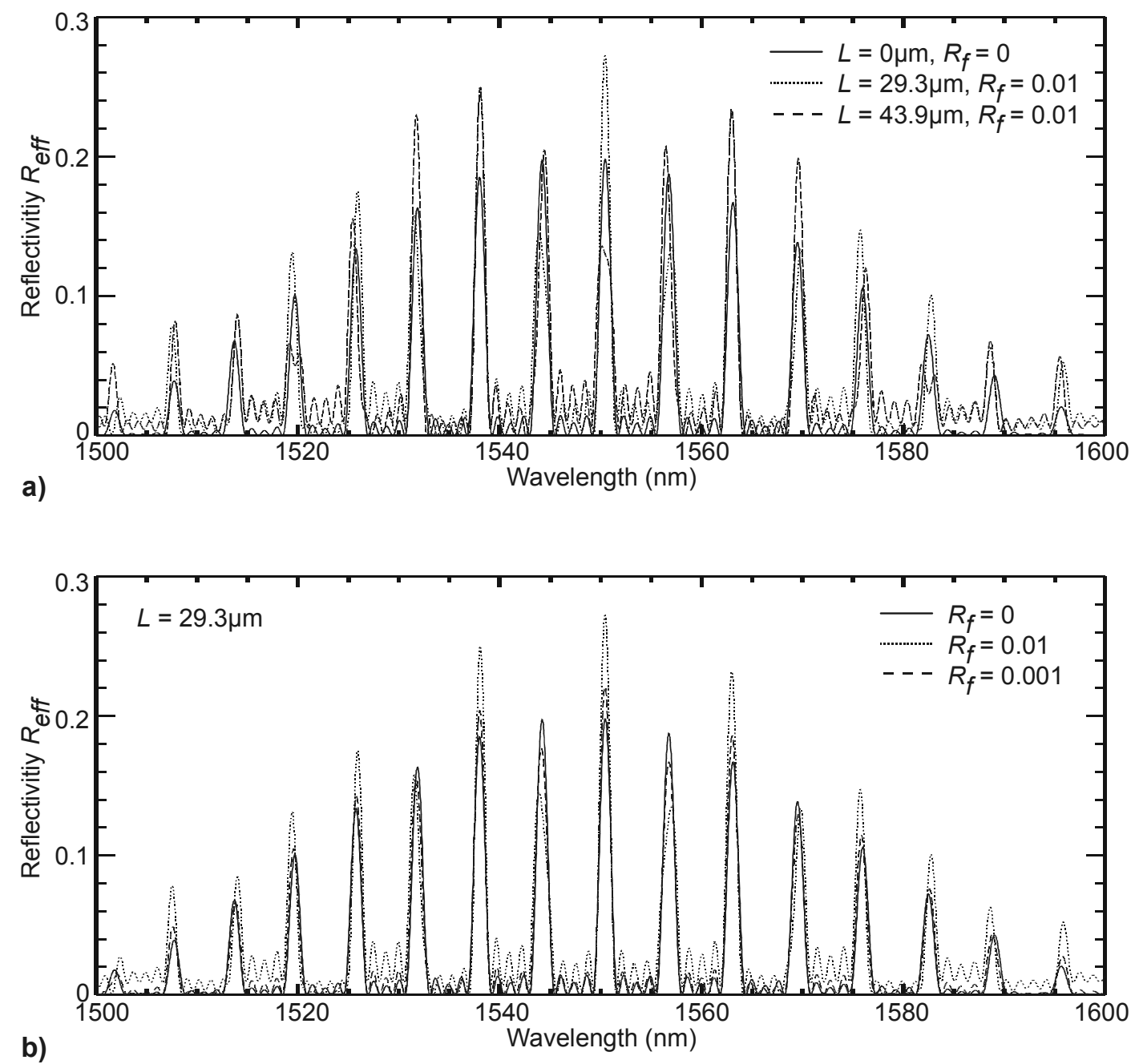

Fig. 4. Reflection spectra of a sampled grating section that interferes with a facet of reflectivity $R_{f}$, which is separated from the sampled grating by a distance $L$.

For large values of $L$, the period of the interference oscillations will become smaller. Therefore, the tendency of getting small shifts of the peak position will become more pronounced. Moreover, frequently also initially single peaks can become split into two peaks. And furthermore, also the amplified spontaneous emission (ASE) noise will impair the device performance. Hence, increasing $L$ for a given value of $R_{f}$ should be avoided at any cost.

On the other hand for small values of $L(<5-10 \mu \mathrm{m})$, the period of the interference oscillation will be reasonably large to obtain an acceptable reflection spectrum. Unfortunately, cleaving the device on both ends with such a high accuracy is technologically very challenging.

Therefore, it appears worth investigating what magnitude of $R_{f}$ is still acceptable to obtain a decent reflection spectrum even for reasonably large values of $L$. Fig. $4 \mathrm{~b}$ illustrates how the reflections spectrum depends on $R_{f}$ for a given value of $L$. As can be seen, even by lowering the facet reflectivity to 0.001 , the distortions in SG reflection spectrum are still fairly pronounced. At this point it should also be taken into account that also the reflection spectrum of the second grating section is subject to distortions brought about by the other facet and that these distortions might add constructively. Although not shown in Fig. 4b, our simulations show that a reflectivity of 0.0001 leaves the SG spectrum practically unaffected. Such a low reflectivity over a wavelength range of several tens of nm can for example by obtained by multilayer AR-coatings, ${ }^{11}$ tilted waveguides ${ }^{12}$ or window structures ${ }^{13}$ or combinations thereof. 


\section{FABRICATION}

The widely tunable twin-guide lasers have been manufactured using the GaInAsP/InP material system. The five epitaxial growth steps that were necessary for the device fabrication have been carried out in a Varian Modular Gen II chemical beam epitaxy (CBE) system and in an Aixtron AIX 200/4 metal-organic vapor phase epitaxy (MOVPE) system.

The fabrication process of the TTG laser is in large parts compatible with standard processing technology used for buried heterostructure lasers. However, some differences arise from the rather unconventional lateral current injection scheme. Before reaching the active and tuning layer, the electron current has to pass through a long $n$-InP channel (see Fig. 5), which is sandwiched between $p$-InP layers. The corresponding interfaces between $p$ - and $n$-InP form forward biased $p$ - $n$-homojunctions under normal device operation. Moreover, the current confinement of this structure is solely due to the differences in bandgaps of active and tuning layer and the surrounding InP and, therefore, the quality of the InP $p$ - $n$-homojunctions is of uttermost importance. Unfortunately these $p$ - $n$ interfaces also coincide with regrowth interfaces. Any contamination or crystal damage that is incorporated at these interfaces leads to increased recombination and thereby deteriorates the current confinement of the device structure. Besides this, the increased carrier recombination at the InP homojunction results also in an additional heat generation, which counteracts the refractive index change in the tuning layer and further limits the achievable continuous tuning range. Thus, a thorough optimization of the numerous regrowth interfaces is required to achieve an efficient current injection and a large continuous tuning range.

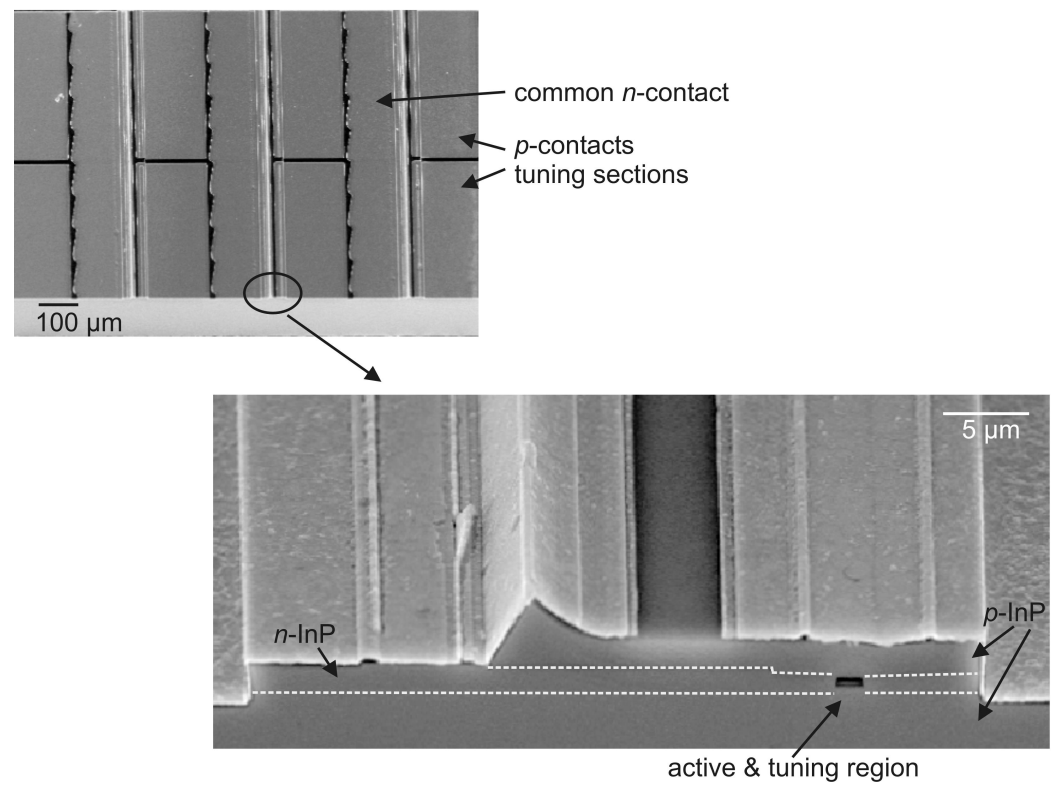

Fig. 5. SEM images showing an SG-TTG laser chip. The dashed white lines in the lower image indicate the location of the InP $p$ - $n$-homojunctions. The common $n$-contact is situated on the left hand side of the ridge and the electron current is injected into the active and tuning regions via the $n$-InP channel. 


\section{TUNING CHARACTERISTICS OF WIDELY TUNABLE TTG LASER DIODES}

The data presented in the following is from $600 \mu \mathrm{m}$ long SG-TTG laser diodes, which comes down to about $300 \mu \mathrm{m}$ of length for each tuning section. Because of technological constraints, these devices were only AR-coated with a quarterwave-thick $\mathrm{Al}_{2} \mathrm{O}_{3}$ film. Thus, the reflectivity of the facet is on the order of 0.01 and accordingly the influence of the facet is not negligible in these devices. For characterization, the lasers were mounted upside up on a $\mathrm{Cu}$ heatsink and were kept at $20^{\circ} \mathrm{C}$ during all measurements.

Fig. 6 shows a typical $L-I$ characteristic. The lasing threshold is reached at $24 \mathrm{~mA}$. Due to the onset of leakage currents across the forward biased backside $p$ - $n$-homojunction, the output power saturates at currents above $100 \mathrm{~mA}$ and reaches a maximum of $12 \mathrm{~mW}$. This saturation behaviour is typical for TTG lasers that do not employ electrical blocking layers.

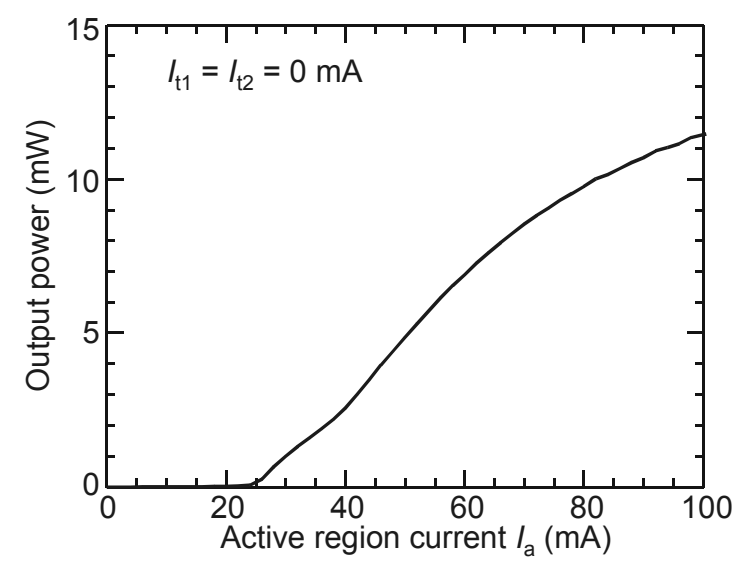

Fig. 6. $L-I$ characteristics of an SG-TTG laser diode.

The tuning behavior of the device is depicted in Fig. 7, showing emission wavelength as well as SMSR as function of the two tuning currents $I_{\mathrm{t} 1}$ and $I_{\mathrm{t} 2}$. From these plots one can easily recognize four supermodes. These are characterized by a high SMSR, which remains between 25 and $37 \mathrm{~dB}$ over the whole tuning range. Furthermore, it is worth mentioning that also a fifth supermode is addressable, but not visible in Fig. 7 since a very precise adjustment of the tuning currents is necessary. A compilation of spectra from the five different supermodes is shown in Fig. 8. For each supermode, one spectrum of the upper and lower limit of the supermode is depicted. The continuous tuning range of the supermode, which is varying between 0.45 and $1.5 \mathrm{~nm}$, is indicated above each supermode. As can be seen, the overall
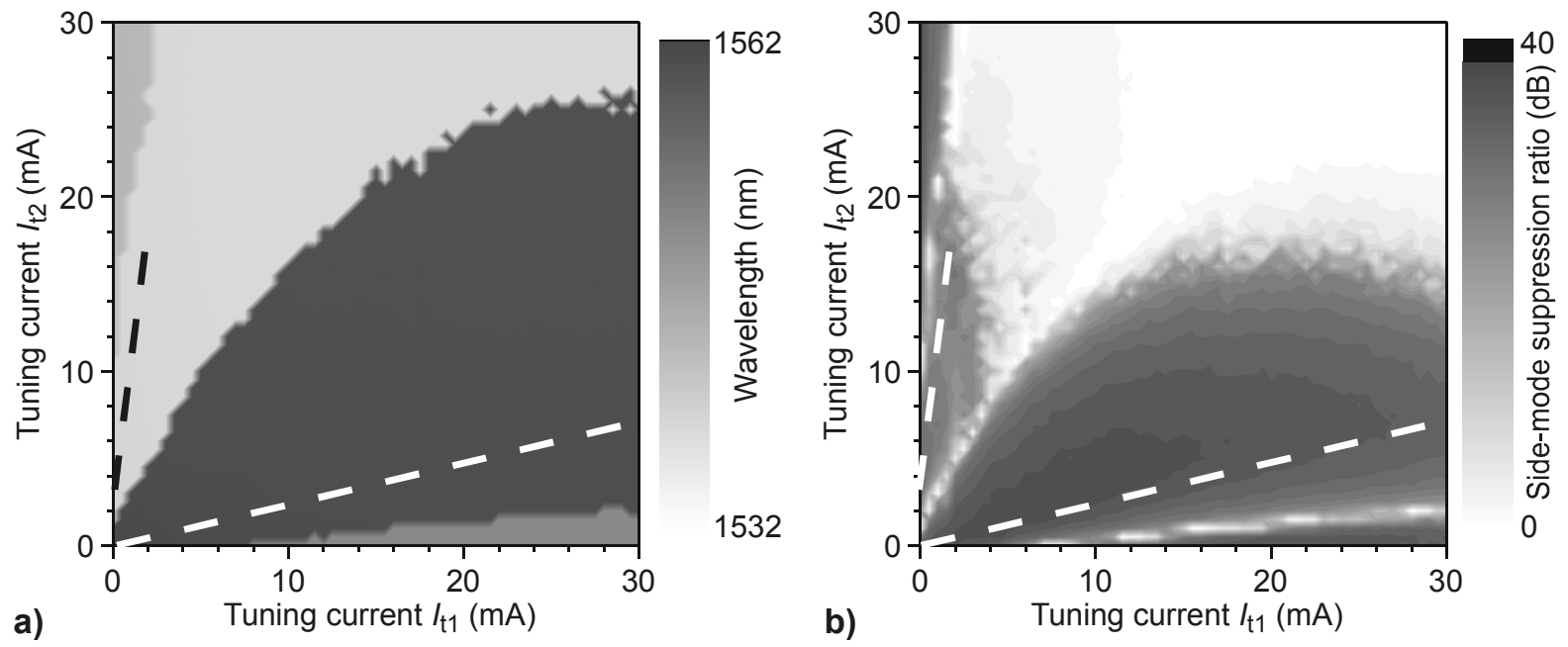

Fig. 7. Tuning behavior of emission wavelength (a) and SMSR (b) of an SG-TTG laser. The dashed lines indicate the tuning curves shown in Fig. 9. 


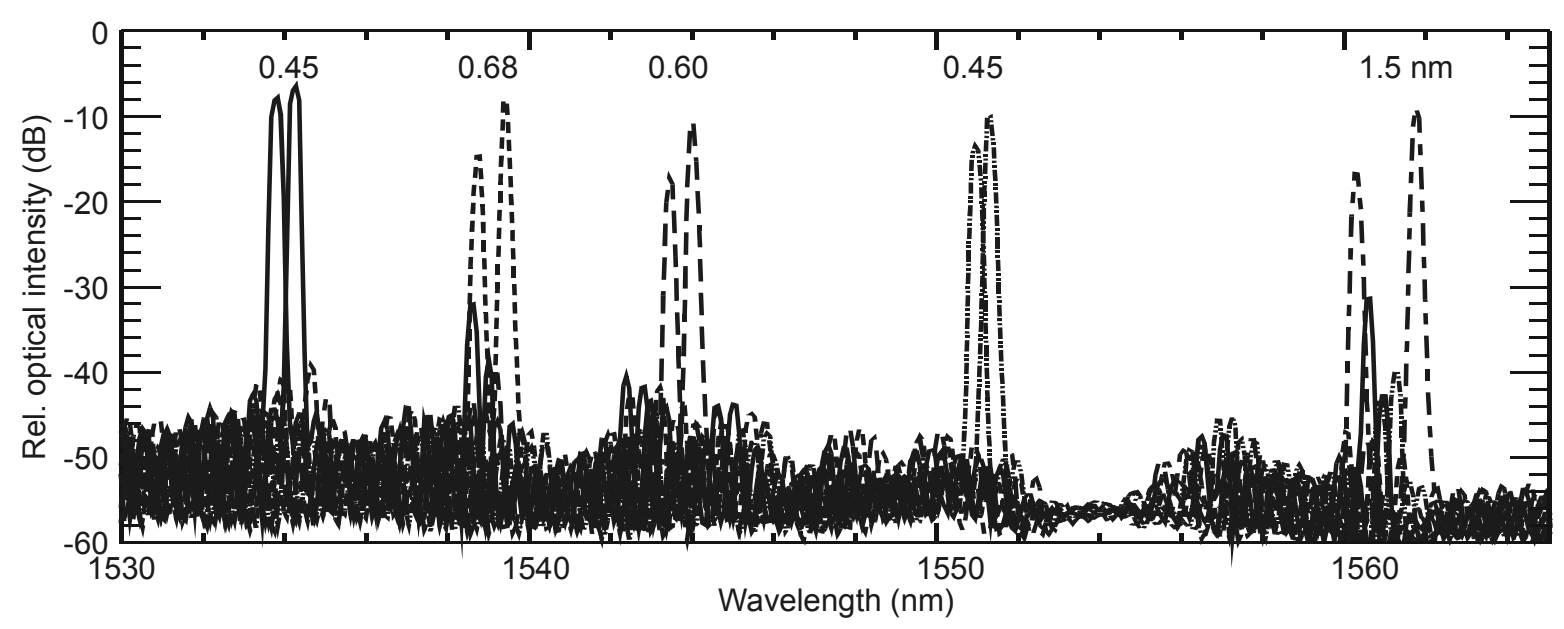

Fig. 8. Spectra showing the upper and lower limits of the five supermodes. The continuous tuning range of each supermode is indicated in the upper part of the image.

tuning range of the SG-TTG laser diode amounts to $28 \mathrm{~nm}$ (from 1534 to $1562 \mathrm{~nm}$ ). However, full wavelength coverage is not possible with these devices, because the continuous tuning range is presently still rather limited. A maximum continuous tuning of $1.5 \mathrm{~nm}$ has been obtained from the present devices. These results indicate that the tuning efficiency is most probably impaired by leakage currents across the frontside $p$ - $n$-homojunction. The leakage currents lead to an undesirable heat generation in the vicinity of the tuning region that counteracts the refractive index change due to the plasma effect. Ultimately this leads to a smaller tuning range. Hence, there is still significant potential for an improvement of the continuous tuning range by which the reflection spectra can be shifted. With an optimised fabrication process, a continuous tuning range of $6-7 \mathrm{~nm}$ is achievable, which is expected to result in full wavelength coverage of the whole tuning range.

Without any tuning currents applied, single-mode emission at a wavelength of $1561.6 \mathrm{~nm}$ with an SMSR of $32 \mathrm{~dB}$ is observed. This is also the supermode, where the central reflection peaks of the SG are lined up, and therefore it exhibits the largest continuous tuning range of all supermodes. The variation of wavelength and SMSR of this main supermode is illustrated in Fig. 9 along with data of a second supermode. As can be seen, the tuning behavior within a supermode is very regular and truly continuous. Only slight variations of the SMSR are observed.
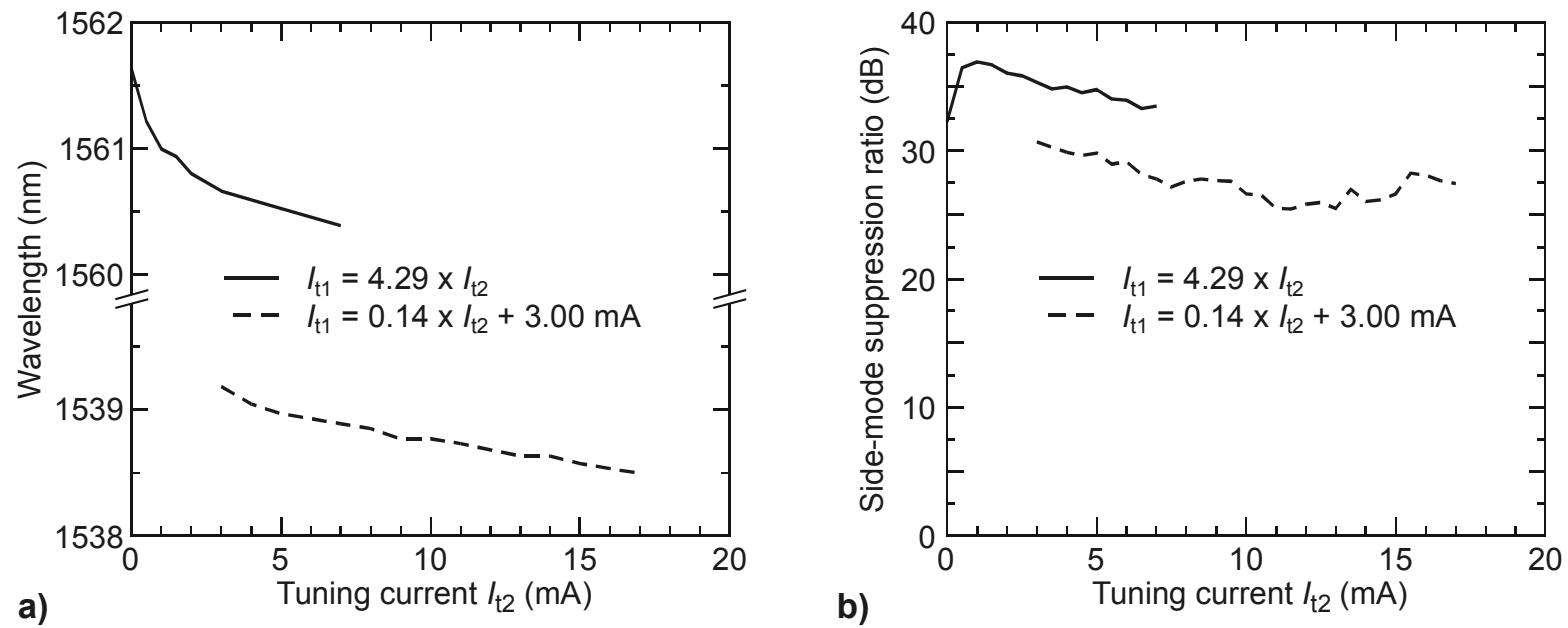

Fig. 9. Variation of emission wavelength (a) and SMSR (b) during continuous tuning (for two selected supermodes, shown as dashed lines in Fig. 7). 


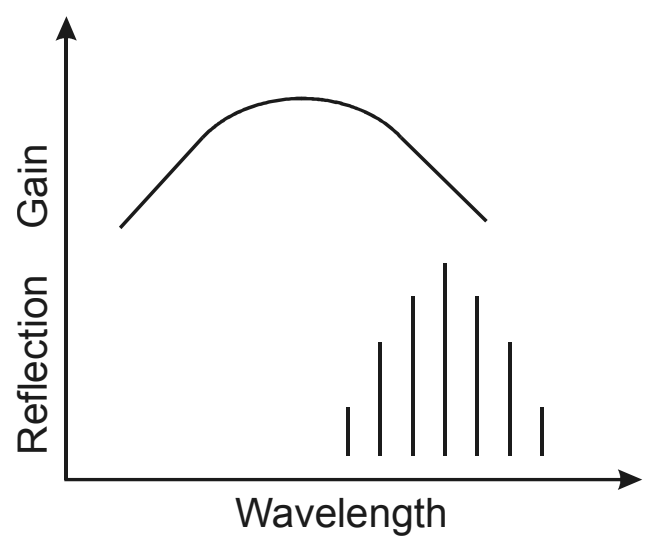

Fig. 10. Detuning of gain peak and SG reflection spectrum.

The supermode hops take place fairly regular. However, one has to mention that the grating center wavelength $(1562 \mathrm{~nm})$ and the gain peak $(1535 \mathrm{~nm})$ are not aligned. This situation is schematically depicted in Fig. 10. With increasing tuning currents, supermode hops only take place towards shorter wavelength or towards the gain peak, respectively. Essentially, the detuning of grating center wavelength and gain peak balances to some extent the difference of gain and mirror losses for higher order reflection peaks that are situated closer towards the gain maximum (i.e. on the left hand side of the reflection peak maximum). Of course, the opposite is true for higher order reflection peaks that are situated further away from the gain maximum (i.e. on the right hand side of the reflection peak maximum).

Furthermore it can be seen from Fig. 8 that the supermode, to be located at $1556 \mathrm{~nm}$, is missing. This is most likely caused by residual facet reflections. Although strong ASE, which is typically observed at any supermode location, is also observed at around $1556 \mathrm{~nm}$, lasing could not be obtained at this wavelength. Instead the supermode hops from $\sim 1561 \mathrm{~nm}$ directly to $\sim 1551 \mathrm{~nm}$. Taking into account the still fairly high reflectivity $(\sim 1 \%)$ of the AR-coated facets, this behaviour is not surprising.

\section{CONCLUSIONS}

The first generation of widely tunable twin-guide laser diodes has clearly proven the design concept of the SG-TTG laser. Wide wavelength tuning over a $28 \mathrm{~nm}$ wavelength range (from 1534 to $1562 \mathrm{~nm}$ ) along with high SMSR has been demonstrated with only two tuning currents.

The overall device performance is presently still impaired by the rather small tuning efficiency of the tuning region as well as by the residual facet reflections. Further technological refinements in order to improve the tuning efficiency and to further minimize facet reflections are expected to result in full wavelength coverage and a regular tuning behaviour.

\section{ACKNOWLEDGMENTS}

This work has been funded by the European Union via the Information Society Technologies (IST) project IST-2000-28244 "NEWTON". Reinhard Laroy acknowledges the Institute for the Promotion of Innovation by Science and Technology in Flanders (IWT) for a specialisation grant. Furthermore, the authors wish to thank Josef Grottenthaler for providing technical assistance during epitaxial growth and device fabrication. 


\section{REFERENCES}

1. F. Delorme: 'Widely Tunable 1.55- $\mu \mathrm{m}$ Lasers for Wavelength-Division-Multiplexed Optical Fiber Communications', IEEE J. Quantum Electron., vol. 34, pp. 1706 - 1716, 1998.

2. P. Werle: 'A review of recent advances in semiconductor laser based gas monitors', Spectrochim. Acta Part A, vol. 54, pp. $197-236,1998$.

3. C.C. Chan, W. Jin, H.L. Ho, and M.S. Demokan: 'Performance Analysis of a Time-Division-Multiplexed Fiber Bragg Grating Sensor Array by use of a Tunable Laser Source’, IEEE J. Sel. Top. Quantum Electron., vol. 6, pp. $741-749,2000$.

4. S.H. Yun, G.J.Tearney, B.E. Bouma, B.H. Park, and J.F. de Boer: 'High-speed spectral-domain optical coherence tomography at $1.3 \mu \mathrm{m}$ wavelength', Opt. Express, vol. 11, pp. 3598 - 3604, 2003

5. M.-C. Amann and J. Buus: 'Tunable Laser Diodes', Artech House, Norwood, USA, 1998.

6. L.A. Coldren: 'Monolithic Tunable Diode Lasers', IEEE J. Sel. Top. Quantum Electron., vol. 6, pp. 988 - 998, 2000.

7. G. Morthier, B. Moeyersoon, and R. Baets: 'A $\lambda / 4$-Shifted Sampled or Superstructure Grating Widely Tunable Twin-Guide Laser’, IEEE Photon. Technol. Lett., vol. 13, pp. 1052 - 1054, 2001.

8. T. Wolf, S. Illek, J. Rieger, B. Borchert, and M.-C. Amann: 'Tunable twin-guide (TTG) distributed feedback (DFB) laser with over $10 \mathrm{~nm}$ continuous tuning range', Electron. Lett., vol. 29, pp. $2124-2125,1993$.

9. V. Jayaraman, Z.-M. Chuang, and L.A. Coldren: 'Theory, Design, and Performance of Extended Tuning Range Semiconductor Lasers with Sampled Gratings', IEEE J. Quantum Electron., vol. 29, pp. 1824 - 1834, 1993.

10. Y. Tohomori, F. Kano, H. Ishii, Y. Yoshikuno, and Y. Kondo: 'Wide tuning with narrow linewidth in DFB lasers with superstructure grating (SSG)', Electron. Lett., vol. 29, pp. 1350 - 1351, 1993.

11. M.C. Farries, J. Buus, M. Kearley: 'Design and fabrication of two layer anti-reflection coatings for semiconductor optical amplifiers', Electron. Lett., vol. 26, pp. 1626 - 1627, 1990.

12. D. Marcuse: 'Reflection Loss of Laser Mode From Tilted End Mirror', IEEE J. Lightwave Technol., vol. 7, pp. 336 $-339,1989$.

13. I. Cha, M. Kitamura, H. Honmou, I. Mito: ' $1.5 \mu \mathrm{m}$ band travelling-wave semiconductor optical amplifiers with window facet structure’, Electron. Lett., vol. 25, pp. 1241 - 1242, 1989. 